EUROPEAN ORGANIZATION FOR NUCLEAR RESEARCH

\author{
CERN - SL Division
}

CERN SL/96-14 (MS)

\title{
THE EFFECTS OF UV RADIATION AND ELECTRON BOMBARDMENT ON THE FLASHOVER CHARACTERISTICS OF ALUMINA BASED HIGH VOLTAGE INSULATORS IN VACUUM.
}

\author{
B. J. Goddard, W. Kalbreier \\ CERN, European Organisation for Nuclear Research,1211 Geneva 23, Switzerland \\ N. S. Xu, R. V. Latham \\ Aston University, Aston Triangle, Birmingham B4 7ET, United Kingdom \\ W. Taylor \\ Farmsum Associates, Brougham Enterprise Centre, Hartlepool, TS24 8EY, United Kingdom \\ D. Chivers \\ AEA Technology, 8 Harwell, Didcot, OX11 0RA, United Kingdom
}

\begin{abstract}
The effects of UV and electron bombardment on the flashover characteristics of highly stressed alumina insulators in vacuum were investigated as part of a project to improve the performance of high voltage insulators in large particle accelerators at CERN. An experimental system has been developed which allowed photon and electron bombardment of stressed insulator samples under vacuum, in order to investigate the causes and characteristics of insulator flashover, and to identify sample preparations which could improve insulator performance.
\end{abstract}

Accepted for the Proceedings of the XVII ${ }^{\text {th }}$ International Symposium on Discharges and Electrical Insulation in Vacuum (ISDEIV XVII), San Francisco, 21-26 July 1996.

Geneva, Switzerland

26 April 1995

SL/Div Reps 


\title{
THE EFFECTS OF UV RADIATION AND ELECTRON BOMBARDMENT ON THE FLASHOVER CHARACTERISTICS OF ALUMINA BASED HIGH VOLTAGE INSULATORS IN VACUUM.
}

\author{
B. J. Goddard, W. Kalbreier \\ CERN, European Organisation for Nuclear Research,1211 Geneva 23, Switzerland \\ N. S. Xu, R. V. Latham \\ Aston University, Aston Triangle, Birmingham B4 7ET, United Kingdom \\ W. Taylor \\ Farmsum Associates, Brougham Enterprise Centre, Hartlepool, TS24 8EY, United Kingdom \\ D. Chivers \\ AEA Technology, 8 Harwell, Didcot, OX11 0RA, United Kingdom
}

\begin{abstract}
The effects of UV and electron bombardment on the flashover characteristics of highly stressed alumina insulators in vacuum were investigated as part of a project to improve the performance of high voltage insulators in large particle accelerators at CERN. An experimental system has been developed which allowed photon and electron bombardment of stressed insulator samples under vacuum, in order to investigate the causes and characteristics of insulator flashover, and to identify sample preparations which could improve insulator performance.
\end{abstract}

\section{INTRODUCTION}

The flashover characteristics of alumina $\left(\mathrm{Al}_{2} \mathrm{O}_{3}\right)$ insulators in vacuum can be modified by irradiation, either in the form of photons or elementary particles, in particular electrons (e-).

In the Large Electron Positron (LEP) collider at CERN, the incidence of high voltage breakdown in the parallel plate separators has been observed to increase with beam current and energy [1], leading to an unacceptable performance degradation which, under certain circumstances, could threaten future operation. Flashover of the alumina insulators is suspected as the main mechanism leading to breakdown [2].

The fundamental causes of such flashover events have been investigated in a laboratory test facility at Aston which allows the bombardment of an insulator surface with UV photons and electrons. The facility also allows comparison of potential technological solutions which might improve insulator performance. To this end, three types of samples were analysed; untreated alumina, samples which had been ion implanted at Harwell with various species and doses, and samples which had been coated using ion beam assisted deposition (IBAD). A detailed description of the comparative performance of the individual proprietary treatments is not within the scope of this paper; however important general trends are reported.

Electrical and optical analysis of the high voltage behaviour were performed for the sample insulators, using a bridged vacuum gap at fields up to $150 \mathrm{kVcm}^{-1}$. Exposure to UV photons of energies up to $5 \mathrm{eV}$ was found to increase the incidence of flashover for all samples, whilst electron charging at energies of $2.5 \mathrm{keV}$ had no noticeable effect. The high voltage behaviour was a strong function of the surface treatment of the insulator; complete suppression of flashover occurred for some samples, associated with linear (Ohmic) prebreakdown I-V characteristics. The results of the test programme are presented, and discussed in the context of the observations made in the actual high voltage devices.

\section{EXPERIMENTAL 2.1 System}

In order to observe the optical activity associated with pre-breakdown and surface flashover processes, an earlier "Transparent Anode" imaging technique [3] was adapted, Figure 1. 


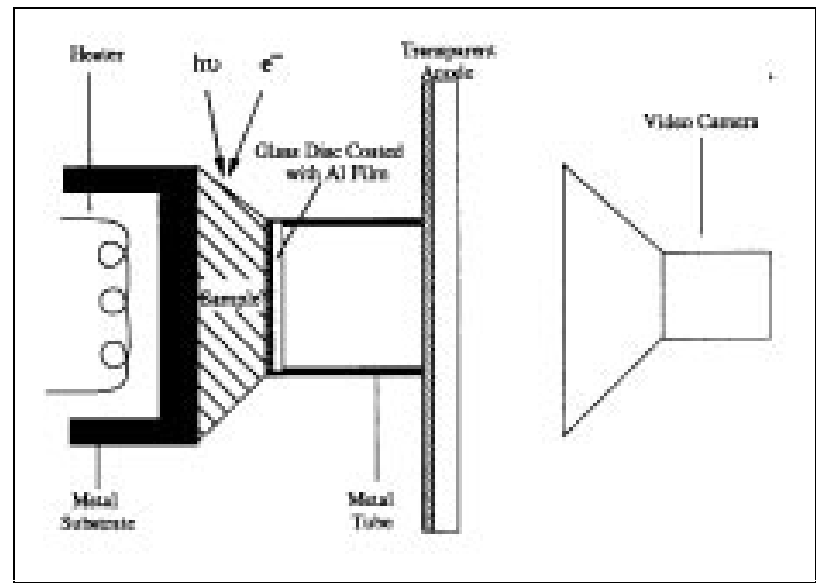

Fig. 1: Schematic description of the transparent anode imaging technique.

A truncated $45^{\circ}$ cone ceramic sample of $20 \mathrm{~mm}$ diameter is sandwiched between two electrodes, i.e. a metal base cathode and a transparent anode assembly. This anode assembly consists of three parts: a glass disc, a metal tube and a large transparent anode that supports one end of the metal tube. The glass disc and the large conducting anode are coated with tin oxide, and all these three parts are electrically connected together. This design of the transparent anode assembly allows direct observation of optical activity occurring both at the slanting surface and in the bulk of a ceramic sample, as may be seen from an optical image shown in Figure 2.

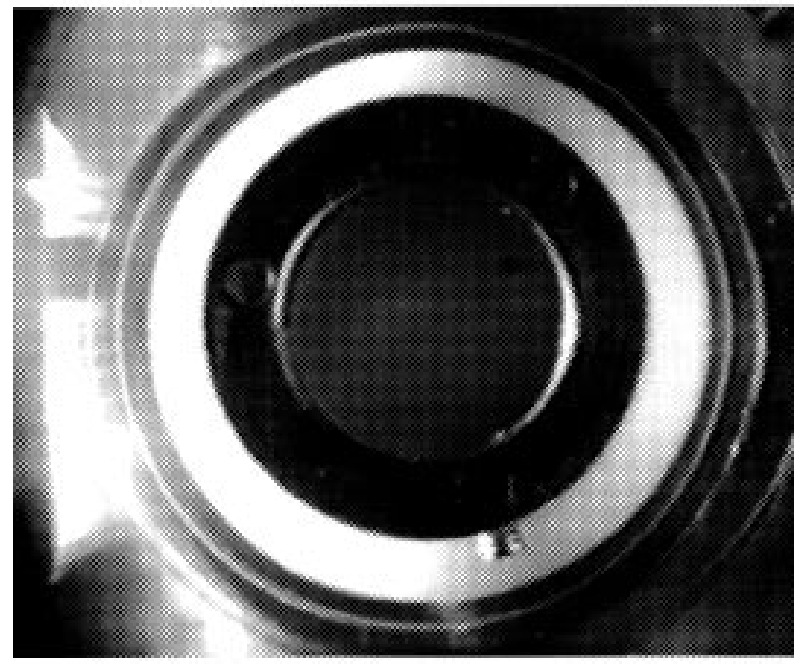

Fig. 2: Inclined surface and bulk of the ceramic sample, viewed through the transparent anode.

As illustrated in Figure 3, in order to subject the surface of a sample to UV radiation, the output from a $100 \mathrm{~W}$ short arc mercury lamp was directed towards half of the slanting surface, through a quartz view port that allowed the transmission of UV photons with maximum energies of $\sim 5 \mathrm{eV}$.

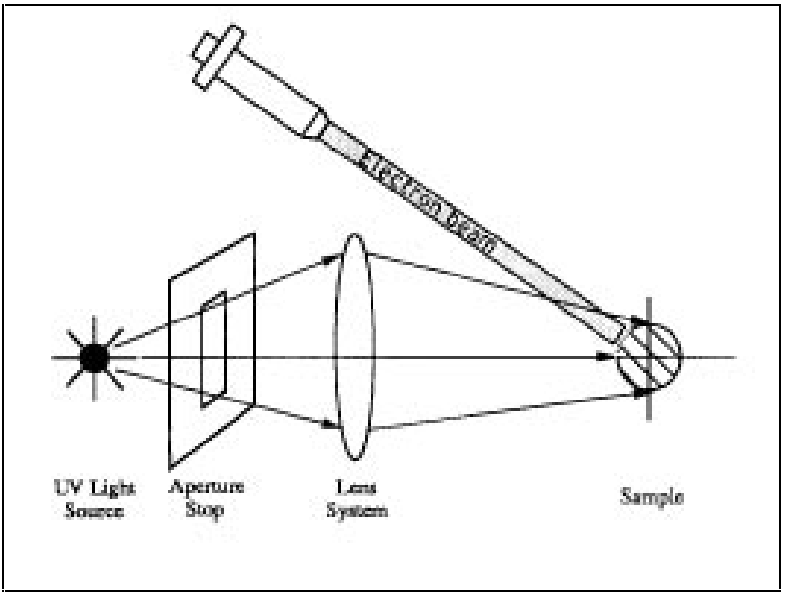

Fig. 3: Schematic arrangement of overlapping electron and UV sample bombardment.

By using an aperture in the beam line, the light only illuminated the slanting surface and its immediate surrounding. In addition, an electron beam from an Omicrom electron gun, of an energy of $2.5 \mathrm{keV}$ and a current of $10 \mu \mathrm{A}$, was arranged to bombard about $25 \%$ of the surface area subjected to UV radiation. Such an arrangement provided an opportunity to examine how UV radiation and implanted charge affect the prebreakdown and flashover behaviour.

\subsection{Sample preparation}

All samples were prepared from a single batch of isostatically pressed sintered alumina (AL300 97\% $\mathrm{Al}_{2} \mathrm{O}_{3}$ ). The CERN production specification [4] for full scale insulators was followed where applicable, including surface grinding to $5 \mu \mathrm{m}$, cleaning and air firing at $1400^{\circ} \mathrm{C}$. The samples were transported in nonfibrous paper envelopes, and handled at all times with surgical gloves. Prior to testing, the sample and electrode assemblies were rinsed with methanol, then allowed to dry naturally. After mounting in the vacuum chamber, the system was pumped to below $10^{-8} \mathrm{mbar}$, before an eight hour bakeout cycle with a four hour plateau at $200^{\circ} \mathrm{C}$. During the tests, the chamber pressure was maintained at better than $10^{-8}$ mbar.

\subsection{Procedure}

The experiment with each sample was carried out by strictly following a standard procedure. This had six steps, in each of which the experimental conditions, in particular the applied radiation conditions, were different. The results were then used to compare the performance of samples processed with different types of surface treatments, and also how the different radiation conditions affected the following important parameters: 
1. Stable inter-electrode current-voltage (I-V) characteristic;

2. Spatial distribution of flashover events; and

3. Flashover frequency (discharge rate);

From the start of each experiment, the video camera was set running to record all associated optical events on film for later detailed examination. The procedure was as follows:

- Step 1: conditioning. Starting with a virgin ceramic, the high voltage was applied across the sample, and increased in $500 \mathrm{~V}$ increments until $29 \mathrm{kV}$. At each voltage, both the stable current flowing between the cathode and anode and the number of sparks were measured. Then the bridged gap was left for conditioning for 30 minutes, during which the number of sparks and the location of their occurrence were also recorded.

- Step 2: no radiation. The stable inter-electrode I-V characteristic was first measured. Subsequently, a 10 minute observation was made with the gap voltage constant at $25 \mathrm{kV}$, followed by a further 20 -minute period at a gap voltage of $28 \mathrm{kV}$.

- Step 3: UV only. The measurements described for step 2 were repeated, but with the sample subjected to the UV light beam illuminating half of the slanting surface area during the whole period.

- Step 4: UV and e-. Prior to measurements, an electron beam of energy of $25 \mathrm{keV}$ and current of $10 \mathrm{~mA}$ bombarded an area of the slanting surface for 10 minutes. The UV light source was then switched on, and the measurements described for step 2 were repeated.

- Step 5: e- only. This was identical to step 4 above, except that the UV light source was not used.

- Step 6: no radiation. Step 6 repeated exactly the measurement of Step 2, in order to see how the radiation processes affect the performance of the sample, and to check that there had been no further conditioning.

\section{RESULTS}

\subsection{Inter-electrode pre-breakdown I-V characteristic}

For untreated $\mathrm{Al}_{2} \mathrm{O}_{3}$ ceramic samples, a typical pre-breakdown I-V characteristic is shown in Figure 4. It exhibits a non-linear property, with strong current increase from $\sim 20 \mathrm{kV}$. The characteristic was not affected by bombardment with UV photons or electrons.

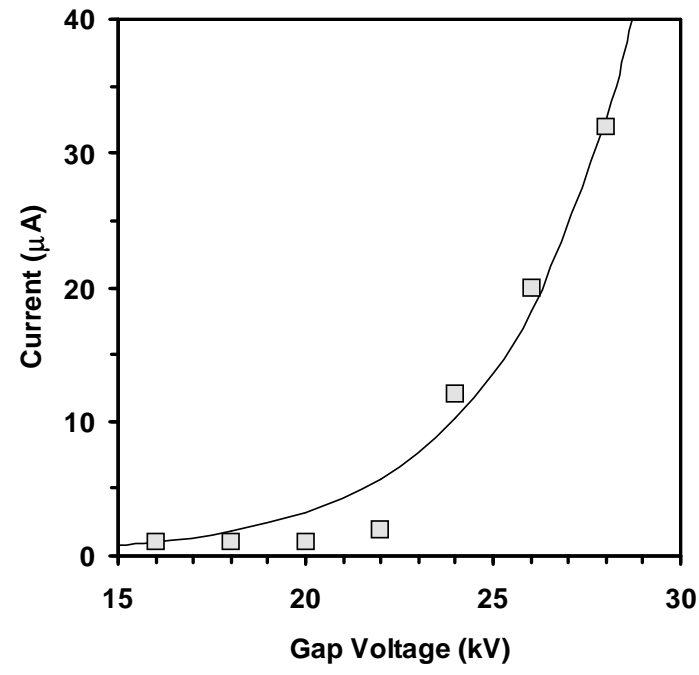

Fig. 4: Non-linear I-V characteristic for untreated alumina sample.

For the ion implanted samples, the characteristic varied with the type of treatment. The currents were lower, at a few $\mu \mathrm{A}$ only, but the non-linear form remained similar to that of an untreated sample. Again, no influence was found from the UV or electron beam.

For the IBAD samples, the I-V characteristic was generally linear (Ohmic), with a much higher current at $28 \mathrm{kV}$, Figure 5 . The UV or electron beam had no effect.

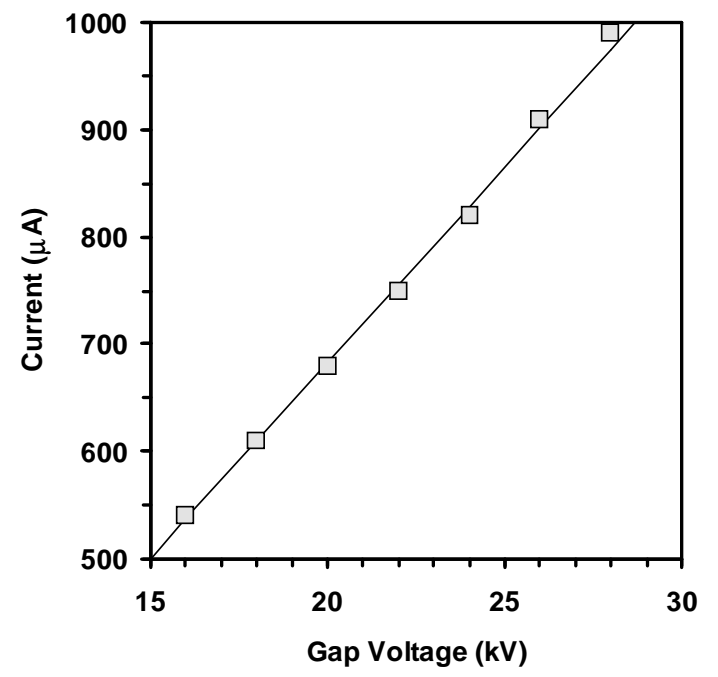

Fig. 5: Linear (Ohmic) I-V characteristic for IBAD treated alumina sample.

\subsection{Spatial distribution of flashover events}

An important general finding is that a surface flashover event is often associated with local optical emission across the gap, Figure 6. This suggests that a surface flashover is a very localised process. This observation is consistent with earlier findings [5], where four types of patterns of optical image that are 
associated with localised surface flashover events are reported.

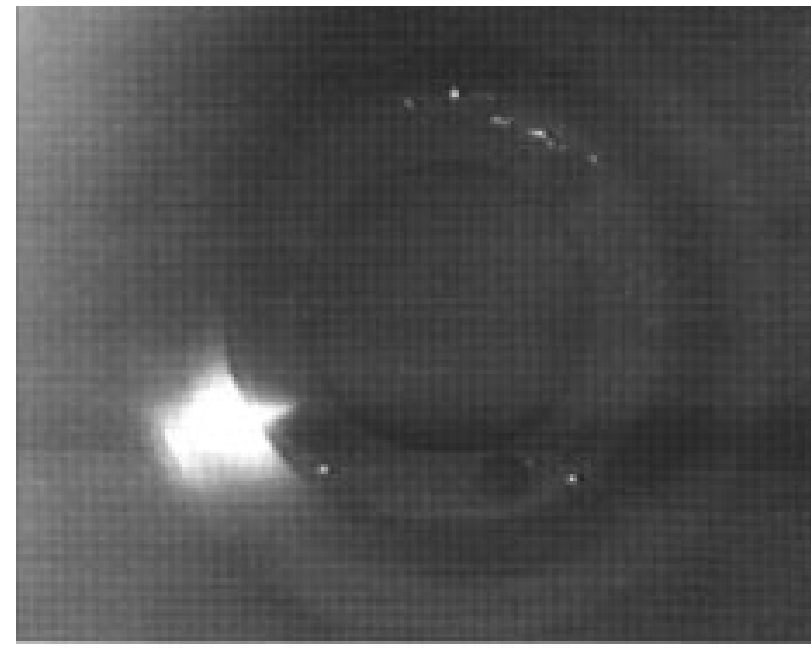

Fig. 6: Localised optical activity (bottom left), associated with a flashover event.

It is noticeable that the pattern of the optical image appears like a fan, with its narrow end at the cathode triple junction, indicating that the flashover was initiated from a cathode triple point.

For all three sample types the illumination of the sample with UV increased the number of flashovers in the illuminated area. This effect was, however, difficult to quantify, as the visible light emitted by the UV lamp prevented most flashover sites from being identified.

\subsection{Flashover frequency (discharge rate)}

In the following, results for the applied voltage of $28 \mathrm{kV}$ (a field of $140 \mathrm{kVcm}^{-1}$ ) are given. Errors quoted are purely statistical.

For untreated $\mathrm{Al}_{2} \mathrm{O}_{3}$ ceramic samples, flashover frequencies after conditioning were typically $28 \pm 9$ per hour. Illumination with UV increased this to $141 \pm 21$ per hour. No effect was seen from e- beam charging.

For the ion implanted samples, flashover frequencies varied considerably, according to the treatment. The best treatments approached zero flashovers per hour; with $64 \pm 14$ flashovers per hour for the worst treatments. UV illumination was found to increase the flashover rate, to $7 \pm 5$ per hour for the best treatment, and $168 \pm 22$ per hour for the worst treatment, Again, no influence was found from ebeam. From this, it is evident that the worst ion implanted samples were similar to the untreated samples, and that the best treatments could improve the performance considerably.

For the IBAD samples, in general the flashover frequencies were very low or zero. UV illumination increased this rate to a maximum of $16 \pm 7$ per hour, although in most cases the flashover remained suppressed. No effect was observed from the e- beam.

\begin{tabular}{|l|llll|}
\hline \multicolumn{1}{l}{} & No radiation & UV & e- & UV + e- \\
\hline Untreate & $13 \pm 6$ & $141 \pm 21$ & $17 \pm 7$ & $231 \pm 26$ \\
d & & & & \\
Implant & 0 & $7 \pm 5$ & 0 & $6 \pm 4$ \\
IBAD & 0 & 0 & 0 & 0 \\
\hline
\end{tabular}

Table 1: Summary of radiation effects. Note that the best of the ion implanted treatments is detailed, with the other treatments giving worse results, in some cases as bad as untreated samples.

Generally, the UV photon irradiation significantly increased spark rates of all samples, whilst electron beam charging had no effect, either alone or in conjunction with the UV. As shown in Figure 7, spark rates were many times higher when an untreated sample was subjected to UV radiation.

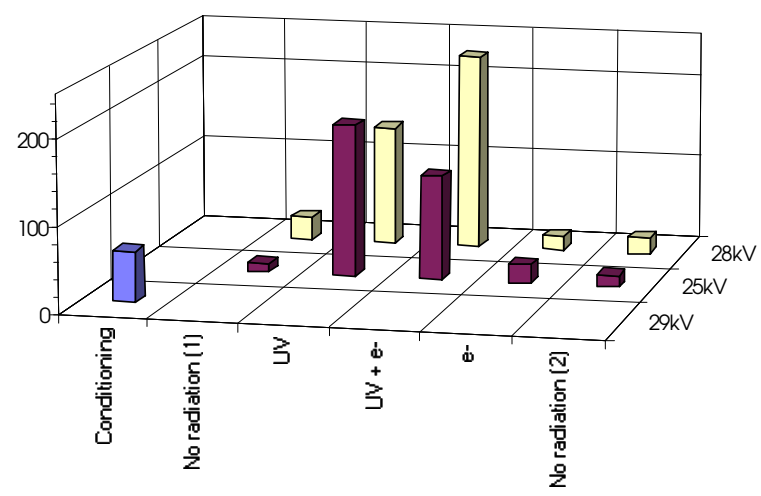

Fig. 7: Spark rates per hour on an untreated sample at $25 \mathrm{kV}$ and $28 \mathrm{kV}$ for different radiation conditions.

The effect of UV illumination is shown in Figure 8, for all sample treatments. Also of interest is the observation that most treatments lead to a reduction in the flashover frequency in the absence of UV radiation.

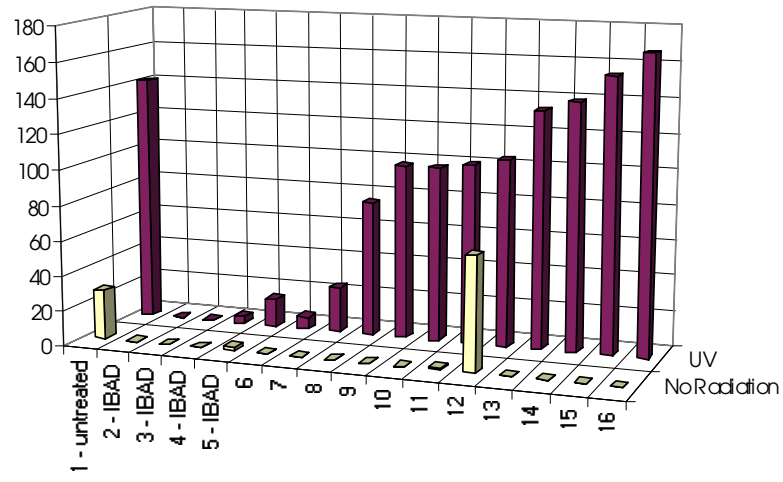

Fig. 8: The effect of UV irradiation on the spark rates per hour at $28 \mathrm{kV}$ for different sample treatments (Number 1 is untreated, 2-5 are IBAD coatings, and 616 are the ion implants).

\section{DISCUSSION}


Irradiation with UV photons has previously been found to induce flashover events on insulator bridged vacuum gaps, see e.g. [6,7]. In addition, it is known that electron charging of an insulator can lead to flashover, even in the absence of an external electric field, see e.g. [8, 9]. In the present study, the dominant effect was from UV irradiation, with electron bombardment having no effect on spark rates, either alone or in conjunction with UV illumination. UV irradiation increased the flashover rate on most sample types, although treatments which gave Ohmic I-V characteristics seemed to suppress flashover under all conditions.

The results indicate that the pre-breakdown and flashover phenomena are intricately related to the surface and near-surface condition of the insulator. The appearance of optical pre-flashover sites has been explained previously as being due to either (a) trapping and detrapping processes [10], or (b) the interaction between electrons emitted from the cathode triple point and the complex charge (electron-hole) distribution in the surface layer [11]. For both models, local plasmas can be triggered by impinging electrons when the density of the charge in this surface layer exceeds some critical value. The role of the UV photons in this process is obviously an important one, and has been attributed to the induced charging of the insulator surface which results from illumination of the system with UV photons [6], either by direct photo-emission or via photoelectrons produced from metallic surfaces. Likewise, the strong polarity effects observed in the LEP separators are qualitatively explained by the clearing effect of positive applied voltage, which effectively prevents photo-electrons from charging the insulator surface.

\section{TECHNOLOGICAL SIGNIFICANCE}

The measurements detailed above were used to select surface treatments to apply to full-scale prototypes for tests in LEP. However, the differences between these tests and operation in LEP should not be underestimated: for the LEP prototype the vacuum chamber pressure is maintained below $10^{-10} \mathrm{mbar}$; the bakeout comprises an eight day cycle with a six day plateau at $300^{\circ} \mathrm{C}$; the energy of the incident photons, will roughly approximate to the classic synchrotron radiation spectrum, which has approximately half of the radiated power at energies above $500 \mathrm{keV}$; the surface area of the insulator is a factor of $~ 200$ larger than the samples; the peak fields are $\sim 7$ times lower; the applied high voltage will reach $220 \mathrm{kV}$.

Although the IBAD treatments seemed to suppress flashover completely under the experimental conditions, the I-V characteristic showed Ohmic behaviour, with a typical resistance of $30 \mathrm{M} \Omega$. Extrapo- lating this to the geometry of the prototype pieces gave a resistance of $700 \mathrm{M} \Omega$, which is a factor of at least 10 too low to be acceptable. Therefore, the best of the ion implantation processes has been applied to full scale pieces, and will be tested in prototype separators in LEP in the 1996 run. The results will provide useful information on the performance of the insulators under real machine conditions.

\section{CONCLUSIONS}

The experimental results presented here demonstrate that the flashover frequency of an insulator bridged vacuum gap under DC stress can be significantly increased by irradiation of the insulator surface and neighbouring electrodes with UV radiation. Treatment of the insulator surface by IBAD coating or ion implantation can significantly alter the performance of the sample, to an extent where flashover is virtually suppressed at fields of $150 \mathrm{kVcm}^{-1}$. Charging of the sample via a $2.5 \mathrm{kV}, 10 \mu \mathrm{A}$ electron beam had no effect.

Flashover events were associated with strong optical pre-breakdown activity, with many flashovers seeming to originate at the cathode triple junction.

The observations indicate that the surface of the insulator plays a critical role in the insulator performance, and that this can be modified by advanced surface engineering techniques. The results of this study are presently being applied to the development of full-scale prototype alumina based insulators for accelerator devices, and first results are expected in the second half of 1997. The technique provides useful information on the mechanisms leading to insulator flashover, with the possibility of further refinement, for example spectroscopic analysis, fast time resolved optical and electrical studies.

\section{ACKNOWLEDGMENTS}

The authors would like to express their gratitude to I.Balchin, H.Freeman and N.Garrel for their valuable contributions throughout the course of this project.

\section{REFERENCES}

[1] W.Kalbreier and B.Goddard, IEEE Trans. Elec. Insul., 28 (4), 444 (1993).

[2] N.Garrel, B.Goddard, W.Kalbreier and R.Keizer, Le Vide: Science, technique et applications, Suppl. to 275, 386 (1995).

[3] R.V.Latham, K.H.Bayliss and B.M.Cox, J. Phys. D: Appl. Phys., 19, 219 (1986). 
[4] N.Garrel, B.Goddard, R.Guinand, W.Kalbreier, R.Kiezer, CERN SL/Tech. Spec. 94-02 (1994).

[5] R.V.Latham, A.Abbot, I.D.Chalmers and N.S.Xu, J. Phys. D: Appl. Phys., 27, 312, 1994.

[6] C.L.Enloe and R.M.Gilgenbach, Plasma Chem. and Plasma Processing, 7 (1), 89 (1987).

[7] P.J.Turchi and W.L.Baker, J.Appl. Phys. 44 (5), 4936 (1973).

[8] G.Blaise and C.Le Gressus, J.Appl. Phys. 69 (9), 6334 (1991).

[9] C.Le Gressus, F.Valin, M.Henriot, M.Gautier, J.P.Duraud, T.S.Sudarshan, R.G.Bommakanti, G.Blaise, J.Appl.Phys 69 (9), 6325 (1991).

[10] B.M.Coaker, N.S.Xu, F.J.Jones and R.V.Latham, J. Phys. D: Appl. Phys., 27, 1448, 1994.

[11] C.L.Enloe and R.M.Gilgenbach, IEEE Trans. Plasma Sci., 16 ( 3), 379 (1988). 